\title{
氮掺杂碳包覆 $\mathrm{Cu}-\mathrm{ZrO}_{2}$ 催化剂的制备及其催化脱氢性能研究
}

\author{
王永胜赵云路赵珍珍兰小林徐金霞 \\ 徐伟祥段正康* \\ (湘潭大学化工学院 环境友好与资源高效利用化工新技术湖南省协同创新中心 湘潭 411105)
}

\begin{abstract}
摘要 本研究以三聚㲵胺作为碳源和氮源, 经高温热解制得具有核壳结构氮掺杂碳 $(\mathrm{CN})$ 包覆的 $\mathrm{Cu}-\mathrm{ZrO}_{2}(\mathrm{CZ})$ 纳米催化 剂( $\mathrm{CZ} @ \mathrm{CN}$ 催化剂), 并研究了铜与三聚㖣胺不同物质的量比对催化剂的影响. 采用扫描电子显微镜( SEM)、透射电子 显微镜(TEM)、 $X$ 射线衍射 $(X R D) 、 X$ 射线光电子能谱 $(X P S) 、 N_{2}$ 物理吸脱附测试 $(B E T) 、 H_{2}$ 程序升温还原 $\left(H_{2}-T P R\right)$ 等 表征技术分析了催化剂的形貌结构及物化性质. 考察了催化剂用于二乙醇胺脱氢的催化性能. 在铜与三聚氰胺物质的 量比为 4: 1 时, 制备的 CZ@CN 催化剂催化活性最高, 亚氨基二乙酸钠收率达 $92.8 \%$, 与普通 CZ 催化剂相比, 反应时 间缩短了 $40 \%$, 催化剂重复使用 8 次后收率依然达到 $88.4 \%$. 结果表明, 适度的 CN 层的引入使催化剂具有更多的 Lewis 碱性位，在脱氢反应中有利于羟基的活化及氢的转移. 同时 $\mathrm{CN}$ 层还可以稳定铜纳米颗粒，提高催化剂稳定性.

关键词 $\mathrm{Cu}-\mathrm{ZrO}_{2}$; 二乙醇胺; 亚氨基二乙酸; 氮掺杂碳; 催化脱氢
\end{abstract}

\section{Study on Preparation of $\mathrm{Cu}-\mathrm{ZrO}_{2}$ Catalyst Coated by Nitrogen-Doped Carbon and Catalytic Dehydrogenation Performance}

\author{
Wang, Yongsheng \\ Zhao, Yunlu \\ Zhao, Zhenzhen Lan, Xiaolin \\ Xu, Jinxia \\ (College of Chenical Engineering, XiangTan University, Hunan Collaborative Innovation Center of New Chemical \\ Technologies for Environmental Benignity and Efficient Resource Utilization, XiangTan 411105)
}

\begin{abstract}
Glyphosate is one of the most widely used herbicides in the world. Current production of glyphosate starts with iminodiacetic acid (IDA). One method of producing IDA starts with the catalytic dehydrogenation of diethanolamine (DEA) using $\mathrm{Cu}-\mathrm{ZrO}_{2}(\mathrm{CZ})$, which is a fairly simple, pollution-free, and cost-effective process. The $\mathrm{Cu}-\mathrm{ZrO} \mathrm{O}_{2}$ catalysts used in this dehydrogenation are fairly efficient and inexpensive, but they tend to agglomerate and inactivate. The development of highly efficient and stable $\mathrm{Cu}-\mathrm{ZrO}_{2}$ catalyst is of great significance. Carbon coated nano-metal particles are a new type of nano-carbon/metal composite materials. Metal materials can be imparted in a small space due to the surface acidity and alkalinity of carbon coated materials and their unique structural characteristics, which is of great significance for the dispersion and oxidation resistance of the loaded nano-metal materials. In this study, melamine was used as a carbon source and a nitrogen source to prepare a $\mathrm{Cu}-\mathrm{ZrO}_{2}$ nanocatalyst $(\mathrm{CZ} @ \mathrm{CN}$ catalyst) coated with nitrogen-doped carbon $(\mathrm{CN})$ with core-shell structure. The effect of different molar ratios of copper and melamine on the catalyst was studied. Scanning electron microscopy (SEM), transmission electron microscopy (TEM), X-ray diffraction (XRD), X-ray photoelectron spectroscopy (XPS), $\mathrm{N}_{2}$ physical adsorption and desorption test $(\mathrm{BET}), \mathrm{H}_{2}$ temperature-programmed reduction $\left(\mathrm{H}_{2}-\mathrm{TPR}\right)$ were used to investigate the morphology and structure of the catalyst. The catalytic performance of the catalyst for the dehydrogenation of diethanolamine was investigated. When the molar ratio of copper to melamine is $4: 1$, the prepared CZ@CN-1 catalyst has the highest catalytic activity. The yield of sodium iminodiacetic acid is $92.80 \%$, and the reaction time is shorter than that of ordinary $\mathrm{CZ}$ catalyst by $40 \%$. The yield of sodium iminodiacetic acid still reaches $88.45 \%$ after reusing 8 times. The results showed that the introduction of the $\mathrm{CN}$ layer makes the catalyst exhibit more Lewis basicity. Meanwhile, it is beneficial to the activation of hydroxyl groups and the transfer of hydrogen in the dehydrogenation reaction. The CN layer can also stabilize copper nanoparticles and improve the stability of the catalyst.

Keywords $\mathrm{Cu}-\mathrm{ZrO}_{2}$; diethanolamine; iminodiacetic acid; nitrogen-doped carbon; transfer dehydrogenation
\end{abstract}

\section{1 引言}

亚氨基二乙酸是一类重要的精细化工中间体，广泛 应用于农业医药水处理等领域. 尤其是作为除草剂草甘
膦的合成原料其使用量巨大，目前草甘膦是世界用量最 大、增长最快的农药产品之一. 二乙醇胺脱氢制备亚氨 基二乙酸的工艺路线具有环保、高效等优点, 其中 $\mathrm{Cu}-\mathrm{ZrO}_{2}$ 催化剂是二乙醇胺脱氢反应中常用的催化剂.

\footnotetext{
*E-mail: dzk0607@163.com; Tel.: 0731-58292456

Received April 10, 2019; published June 12, 2019.

Project supported by the National Natural Science Foundation of China (No. 21576229).

项目受国家自然科学基金(No. 21576229)资助.
} 
铜基纳米材料导电性好, 耐腐蚀、耐压性强且价格 低廉，广泛应用于催化有机转化、电催化和光催化 ${ }^{[1]}$ 等 领域. 但是, 具有催化活性的铜纳米粒子 $(\mathrm{Cu} \mathrm{NPs})$ 在反 应时易团聚甚至烧结, 需要引入载体以增强催化剂的稳 定性 ${ }^{[2]}$. $\mathrm{ZrO}_{2}$ 具有弱酸碱性、良好的热稳定性和较高的 机械强度, 因此 $\mathrm{ZrO}_{2}$ 作为载体被广泛应用于催化剂领 域. 一方面, $\mathrm{ZrO}_{2}$ 作为载体, 可以为得失电子的 $\mathrm{Cu}$ 粒子 提供电荷缓冲, 能有效提高铜基催化剂活性 ${ }^{[3]}$; 另一方 面, $\mathrm{ZrO}_{2}$ 的高机械强度和良好的热稳定性, 可以防止铜 纳米粒子的烧结, 使催化剂的使用寿命增长 ${ }^{[4]}$.

碳包覆纳米金属颗粒是一种新型的纳米碳/金属复 合材料, 以其独特的形态结构和性质已成为最热门的研 究热点之一 ${ }^{[5]}$. 由于碳与金属间温和的相互作用, 不仅 能增强催化剂抗积碳能力, 还能有效提高负载金属的还 原性能; 同时, 碳包覆材料表面酸碱性及其独特的结构 特征, 可以将金属物质禁锢在很小的空间内, 对负载的 金属纳米材料的分散性、抗氧化性有重要意义 ${ }^{[6 ~ 9]}$.

氮掺杂的碳和氮化碳是一类具有重要用途的功能 材料, 可用于电化学、化学催化剂及过渡金属催化剂的 载体 ${ }^{[10,11,12]}$. 氮原子具有高的富电性, 将氮引入碳框架, 会促进表面碱性位点的形成 ${ }^{[13]}$. 另外, 氮原子能够更加 有效地分散和稳定铜粒子, 对于 $\mathrm{N}^{-}$掺杂的还原氧化石 墨烯(NG), 氮原子可以提供丰富的孤电子对和官能团, 为 $\mathrm{Cu}^{2+}$ 提供结合位点, 通过静电相互作用形成 $\mathrm{NG}^{-}$、 $\mathrm{Cu}^{2+}$, 有效吸附铜粒子而避免其发生聚集 ${ }^{[14,15]}$. 将 $\mathrm{Pt}$ 纳 米颗粒嵌入介孔碳或 $\mathrm{N}$ 掺杂的多孔碳/碳纳米管中, 可 以显著影响金属 $\mathrm{Pt}$ 的电子结构, 从而提高其催化活性 和稳定性 ${ }^{[16]}$. 因此, 氮掺杂在催化剂体系中起着重要作 用.

基于此我们提出一种简单有效的策略, 通过引入三 聚氰胺经过简单热解, 制备出氮掺杂碳包覆的 $\mathrm{CZ} @ \mathrm{CN}$ 纳米催化剂, 铜与三聚氧胺物质的量比为 $4: 1 、 2: 1$ 、 $4: 3$ 和 $1: 1$ 分别命名为 $\mathrm{CZ} @ \mathrm{CN}-1 、 \mathrm{CZ} @ \mathrm{CN}-2$ 、 CZ@CN-3 和 CZ@CN-4 并表征其结构, 探究其催化剂 性能. 为制备高效的 $\mathrm{Cu}$ 基脱氢催化剂提供了新的方法 思路.

\section{2 结果与讨论}

\section{$2.1 X$ 射线粉末衍射 $(X R D)$}

对 $\mathrm{CZ}$ 及铜与三聚氰胺不同物质的量比下催化剂样 品进行 XRD 测试, 探究三聚氧胺的用量对产物晶体形 成的影响. 在 $2 \theta=30.2^{\circ} 、 34.7^{\circ} 、 60.9^{\circ}$ 为 $\mathrm{ZrO}_{2}$ 的衍射峰 (JCPDS no. 37-1484), 在 $2 \theta=43.3^{\circ} 、 50.4^{\circ}$ 和 $74.1^{\circ}$ 为 $\mathrm{Cu}$ 的衍射峰, 分别对应的是 $\mathrm{Cu}(111) 、 \mathrm{Cu}(200)$ 和 $\mathrm{Cu}(220)$ 晶面(JCPDS no. 04-0836). 样品中没有出现 $\mathrm{CuO}$ 与 $\mathrm{Cu}_{2} \mathrm{O}$ 的衍射峰, 说明样品被氢气充分还原. 在图中没 有发现氮化碳和石墨碳的衍射峰, 表明碳氮物种的结晶
度低. 另外, 铜与三聚氰胺不同配比会影响 $\mathrm{Cu}$ 的结晶 度，具体表现为: $\mathrm{CZ} @ \mathrm{CN}-2>\mathrm{CZ}>\mathrm{CZ} @ \mathrm{CN}-3>$ $\mathrm{CZ} @ \mathrm{CN}-1>\mathrm{CZ} @ \mathrm{CN}-4$, 说明在铜与三聚氧胺物质的量 比为 $2: 1$ 时有利于高结晶度的 $\mathrm{Cu}$ 生成. 运用 Scherrer 公式计算各样品中 $\mathrm{Cu}$ 粒子的粒径尺寸(表 1). 图(b)为样 品 $\mathrm{CZ}$ 和 CZ@CN-1 在 $150{ }^{\circ} \mathrm{C}$ 空气氛围下焙烧 $2 \mathrm{~h}$ 后的 $\mathrm{XRD}$ 图. 由图可知, 两个样品均在 $2 \theta=38.6^{\circ}$ 处出现 $\mathrm{CuO}(111)$ 的特征衍射峰 (JCPDS no. 05-0661), 其中, $\mathrm{CZ} @ \mathrm{CN}-1$ 样品的 $\mathrm{CuO}$ 的衍射峰最小但 $\mathrm{Cu}(111)$ 的衍射 峰最大, 表明 CZ@CN-1 样品具有良好的抗氧化性.

表 1 催化剂的组成、结构数据

Table 1 Compositions and structure data of catalysts

\begin{tabular}{|c|c|c|c|c|c|c|c|}
\hline \multirow{2}{*}{ Complex } & \multicolumn{3}{|c|}{ Content ${ }^{a} / \mathrm{wt} \%$} & \multirow{2}{*}{$-S_{\mathrm{BET}}^{b} /\left(\mathrm{m}^{2} \cdot \mathrm{g}^{-1}\right)$} & \multirow{2}{*}{$V_{\mathrm{p}}^{\mathrm{c}} /\left(\mathrm{cm}^{2} \cdot \mathrm{g}^{-1}\right.$} & \multirow{2}{*}{$d_{\mathrm{p}}{ }^{\mathrm{d}} / \mathrm{nm}$} & \multirow{2}{*}{$D_{\mathrm{Cu}}{ }^{e} / \mathrm{nn}$} \\
\hline & $\mathrm{Cu}$ & $\mathrm{C}$ & $\mathrm{N}$ & & & & \\
\hline $\mathrm{CZ}$ & 14.6 & & & 22.5 & 0.036 & 3.4 & 23.6 \\
\hline CZ@CN-1 & 14.4 & 19.8 & 2.5 & 28.6 & 0.045 & 3.6 & 11.3 \\
\hline $\mathrm{CZ} @ \mathrm{CN}-2$ & 14.0 & 20.3 & 2.7 & 20.2 & 0.030 & 3.6 & 11.0 \\
\hline $\mathrm{CZ} @ \mathrm{CN}-3$ & 13.7 & 21.0 & 3.0 & 15.4 & 0.024 & 5.1 & 10.5 \\
\hline CZ@CN-4 & 13.3 & 21.9 & 3.1 & 9.6 & 0.019 & 3.4 & 10.9 \\
\hline
\end{tabular}

${ }^{a}$ Determined by elemental analysis. ${ }^{b}$ Specific surface area calculated using the BET method. ${ }^{c}$ Total pore volume. ${ }^{d}$ Mean pore diameter. ${ }^{e}$ Average crystallite size of metallic copper particles based on XRD pattern.
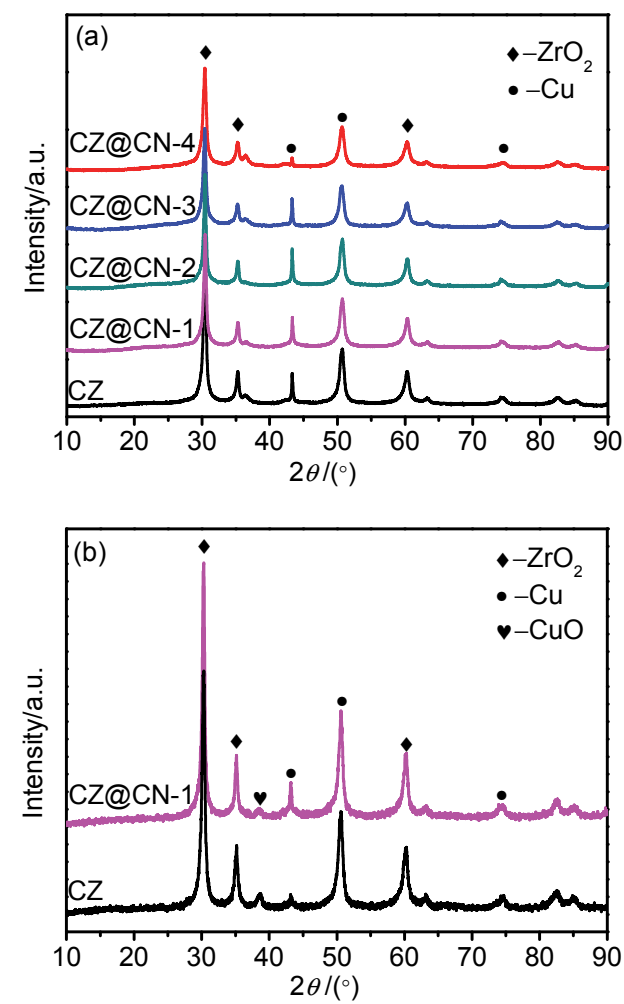

图 1 (a)样品 $\mathrm{X}$ 射线粉末衍射 $(\mathrm{XRD})$ 图; (b)样品 $\mathrm{CZ}$ 和 $\mathrm{CZ} @ \mathrm{CN}-1$ 在 $150{ }^{\circ} \mathrm{C}$ 空气氛围下焙烧 $2 \mathrm{~h}$ 后的 XRD 图

Figure 1 (a) The XRD patterns of the samples; (b) The XRD patterns of $\mathrm{CZ}$ and $\mathrm{CZ} @ \mathrm{CZ}-1$ roasted for $2 \mathrm{~h}$ at $150{ }^{\circ} \mathrm{C}$ 


\section{2 扫描电子显微镜(SEM)}

图 2a 为样品 $\mathrm{CZ}$ 催化剂的 SEM 图, 从图中可以看 出, $\mathrm{CZ}$ 催化剂中 $\mathrm{ZrO}_{2}$ 呈现不规则的片层状结构并且催 化剂尺寸较大. 图 $2 \mathrm{~b} 、 2 \mathrm{c}$ 为样品 $\mathrm{CZ} @ \mathrm{CN}-1$ 催化剂的 $\mathrm{SEM}$ 图. 与 $\mathrm{CZ}$ 催化剂一样表现出 $\mathrm{ZrO}_{2}$ 不规则片层状载 体的形貌, 通过图(c)可以看出 $\mathrm{ZrO}_{2}$ 载体表面负载的 $\mathrm{Cu}$ 纳米粒子. 图 $2 \mathrm{~d} \sim 2 \mathrm{f}$ 分别为铜与三聚氰胺物质的量比 为 $2: 1 、 4: 3$ 和 $1: 1$ 的 $\mathrm{CZ} @ \mathrm{CN}$ 催化剂的 SEM 图. 从 图中可以看出, 随着三聚氧胺添加量逐渐增多, 催化剂 表面的碳层厚度增加, 导致 $\mathrm{ZrO}_{2}$ 不规则片层状载体的
形貌特征逐渐减弱，并且催化剂尺寸越来越小，表明 $\mathrm{CN}$ 层的引入可以使催化剂尺寸减小.

\section{3 透射电子显微镜(TEM)}

为了观察 $\mathrm{CZ} @ \mathrm{CN}$ 催化剂的形貌和微观结构, 对样 品 $\mathrm{CZ} @ \mathrm{CN}-1$ 进行 TEM 表征. 由图可知, 发现粒径约为 $15 \mathrm{~nm}$ 的 $\mathrm{Cu} N P s$ 均匀分散在载体表面. 由图(b)可以看 出明显的铜纳米粒子连续的晶格条纹, 且间距为 0.21 $\mathrm{nm}$ 与面心立方结构金属铜(111)晶面间距一致，进一步 证明了负载的粒子为 $\mathrm{Cu}$ NPs. 更为重要的是 $\mathrm{Cu}$ NPs 被 厚度约为 $3.5 \mathrm{~nm}$ 的氮掺杂的碳薄层所包裹，可以清晰地

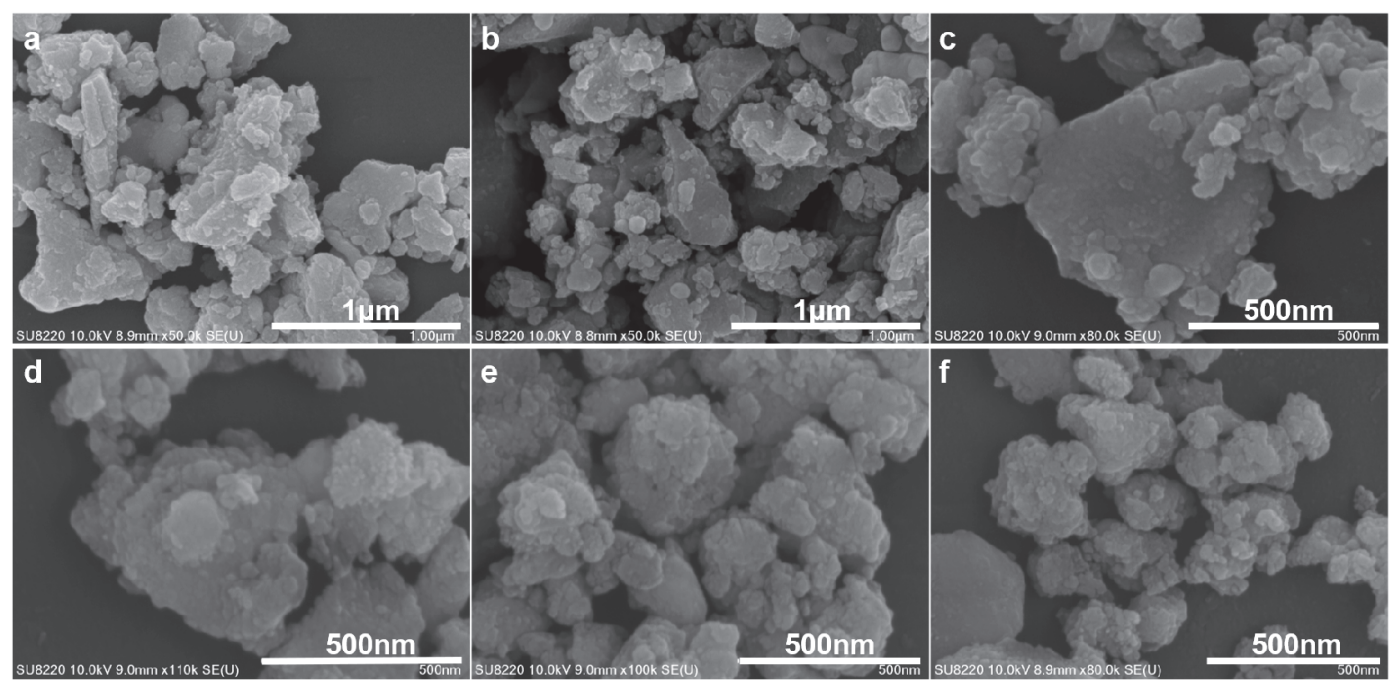

图 2 CZ (a), CZ@CN-1 (b, c), CZ@CN-2 (d), CZ@CN-3(e)和 CZ@CN-4(f)的 SEM 图

Figure 2 SEM images of CZ (a), CZ@CN-1 (b, c), CZ@CN-2 (d), CZ@CN-3 (e) and CZ@CN-4 (f)

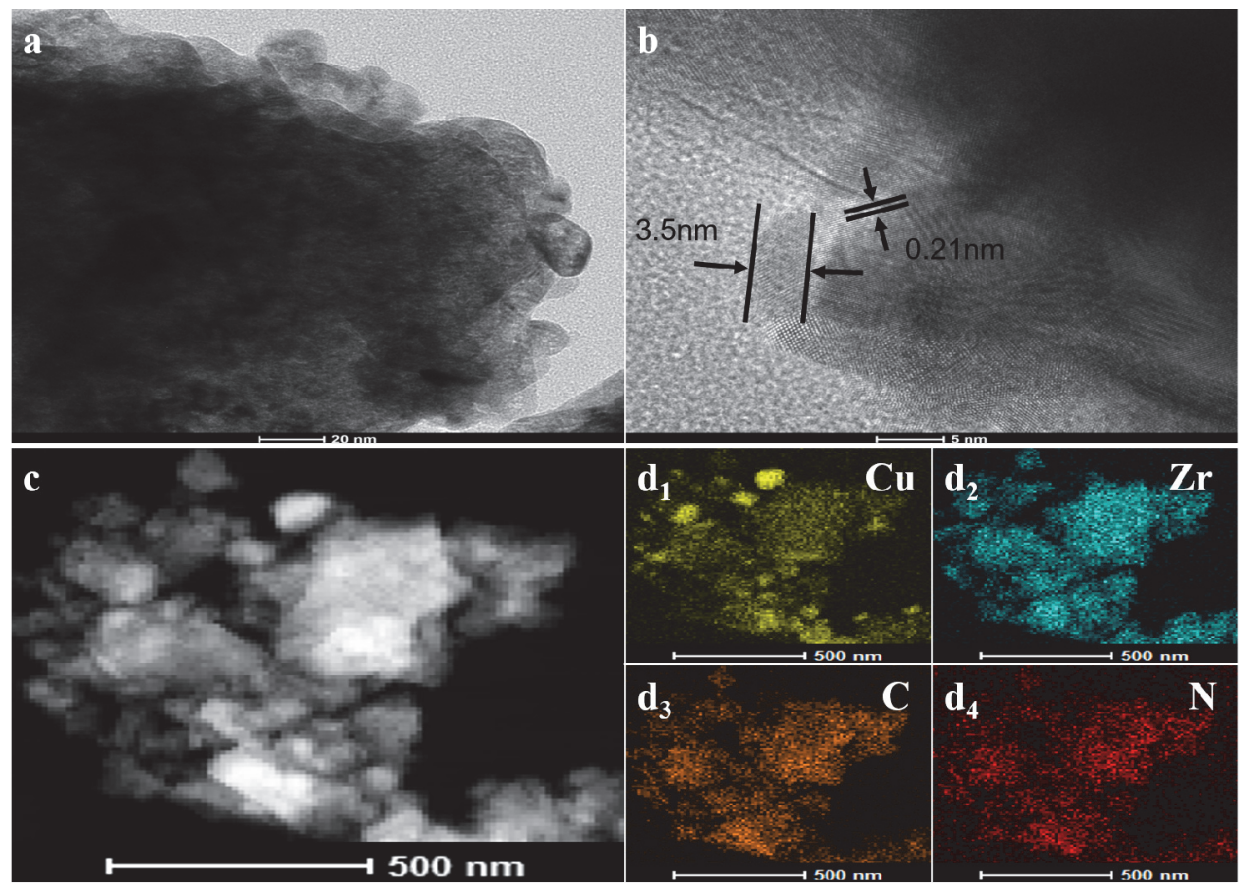

图 3 样品 $\mathrm{CZ} @ \mathrm{CN}-1$ 的 TEM 图(a, b); 高分辨 STEM 图(c) 和 $\mathrm{Cu}\left(\mathrm{d}_{1}\right) 、 \mathrm{Zr}\left(\mathrm{d}_{2}\right) 、 \mathrm{C}\left(\mathrm{d}_{3}\right) 、 \mathrm{~N}\left(\mathrm{~d}_{4}\right)$ 的元素面扫图

Figure 3 The TEM (a, b) images of CZ@CN-1; high-magnification STEM image (c) with EDS mappings of Cu $\left(\mathrm{d}_{1}\right), \mathrm{Zr}\left(\mathrm{d}_{2}\right), \mathrm{C}\left(\mathrm{d}_{3}\right)$ and N $\left(\mathrm{d}_{4}\right)$ elements 
看出 $\mathrm{Cu} \mathrm{NPs}$ 核与碳层之间的差异. 对催化剂进行元素 扫描, 如图 $3 \mathrm{~d}$ 可知, $\mathrm{Cu} 、 \mathrm{Zr} 、 \mathrm{C}$ 和 $\mathrm{N}$ 元素均匀地分布在 催化剂表面, 表明 $\mathrm{C}$ 和 $\mathrm{N}$ 元素成功地引入 $\mathrm{CZ}$ 催化剂体 系中, 制备出具有核壳结构地碳氮包覆的铜纳米催化 剂.

\section{$2.4 \mathrm{~N}_{2}$ 吸脱附曲线(BET)}

采用 $\mathrm{N}_{2}$ 吸脱附实验对样品进行表征并分析了各样 品的比表面积和孔径. 图 $4 \mathrm{a}$ 为样品 $\mathrm{CZ}$ 和不同铜与三聚 氭胺物质的量比的 CZ@CN 催化剂的 $\mathrm{N}_{2}$ 吸附-脱附等温 线. 图 $4 \mathrm{~b}$ 为相应的孔径分布图. 结合表 1 和图 4 可知, 与 $\mathrm{CZ}$ 催化剂相比, $\mathrm{CZ} @ \mathrm{CN}-1$ 催化剂比表面积和孔容较 大, 孔径较小且主要分布在 $3 \sim 4 \mathrm{~nm}$, 结合表 2 可知, 较 大的比表面积和较小的孔径可以使催化剂的性能提高. 随着三聚氭胺添加量的不断增加, 催化剂外层的碳层变 厚, 催化剂的比表面积和微孔体积逐渐下降, 导致催化 剂催化活性降低. 因此, 在 $\mathrm{Cu}-\mathrm{ZrO}_{2}$ 催化剂中引入适量 的三聚氭胺, 形成厚度适中的 $\mathrm{CN}$ 层, 可以有效地增加 催化剂的比表面积.
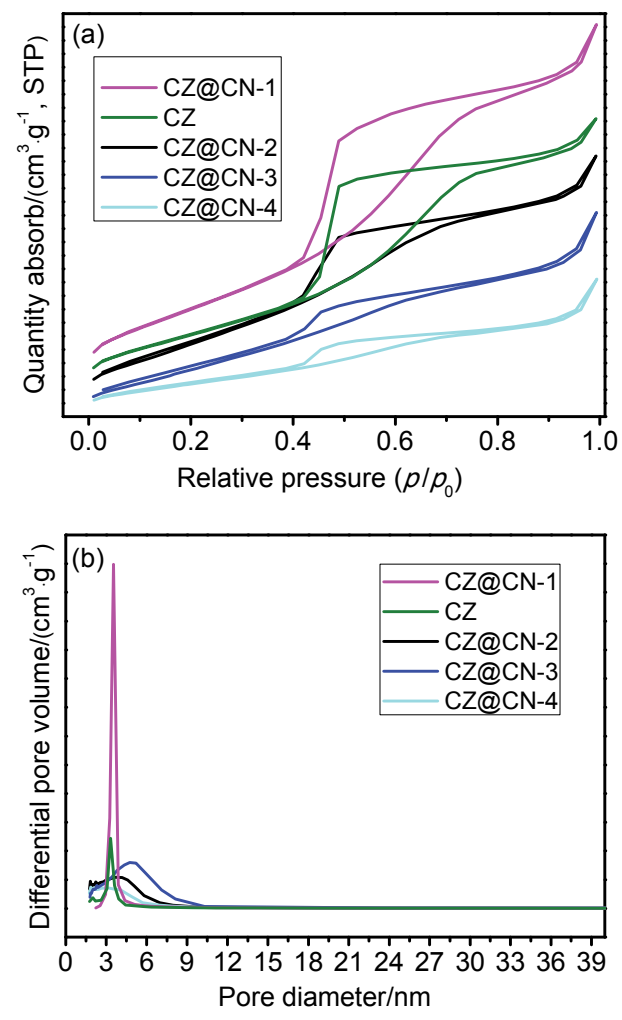

图 4 样品的氮气吸脱附等温线(a)和孔径分布曲线(b)

Figure 4 Nitrogen adsorption-desorption isotherms (a) and pore size distribution curves (b) of samples

\section{$2.5 \mathrm{CO}_{2}$ 程序升温脱附 $\left(\mathrm{CO}_{2}\right.$-TPD)}

为了分析催化剂表面的碱性位, 对五个样品进行 $\mathrm{CO}_{2}$-TPD 的表征, 如图 5a 所示. 五个催化剂样品都呈现 了位于 $140{ }^{\circ} \mathrm{C}$ 的 $\mathrm{CO}_{2}$ 脱附峰, 对应于弱碱性位 ${ }^{[17]}$. 除
表 2 样品用于二乙醇胺脱氢制亚氨基二乙酸钠的性能结果

Table 2 Catalytic performance of samples in the dehydrogenation of diethanolamine

\begin{tabular}{cccccc}
\hline $\begin{array}{c}\text { 催化剂 } \\
\text { 样品 }\end{array}$ & $\begin{array}{c}\text { 催化剂 } \\
\text { 用量/g }\end{array}$ & $\begin{array}{c}\mathrm{DSIDA} \\
\text { 收率/\% }\end{array}$ & $\begin{array}{c}\text { 反应时间/ } \\
\min \end{array}$ & $\begin{array}{c}\text { 初始产气 } \\
\text { 温度/ }{ }^{\circ} \mathrm{C}\end{array}$ & $\begin{array}{c}\text { 初次排气 } \\
\text { 时间 } / \mathrm{min}\end{array}$ \\
\hline $\mathrm{CZ} @ \mathrm{CN}-4$ & 2 & 86.0 & 240 & 110 & 35 \\
$\mathrm{CZ} @ \mathrm{CN}-3$ & 2 & 89.1 & 180 & 110 & 35 \\
$\mathrm{CZ} @ \mathrm{CN}-2$ & 2 & 90.3 & 120 & 110 & 30 \\
$\mathrm{CZ} @ \mathrm{CN}-1$ & 2 & 92.8 & 90 & 90 & 25 \\
$\mathrm{CZ}$ & 2 & 92.3 & 150 & 130 & 30 \\
\hline
\end{tabular}
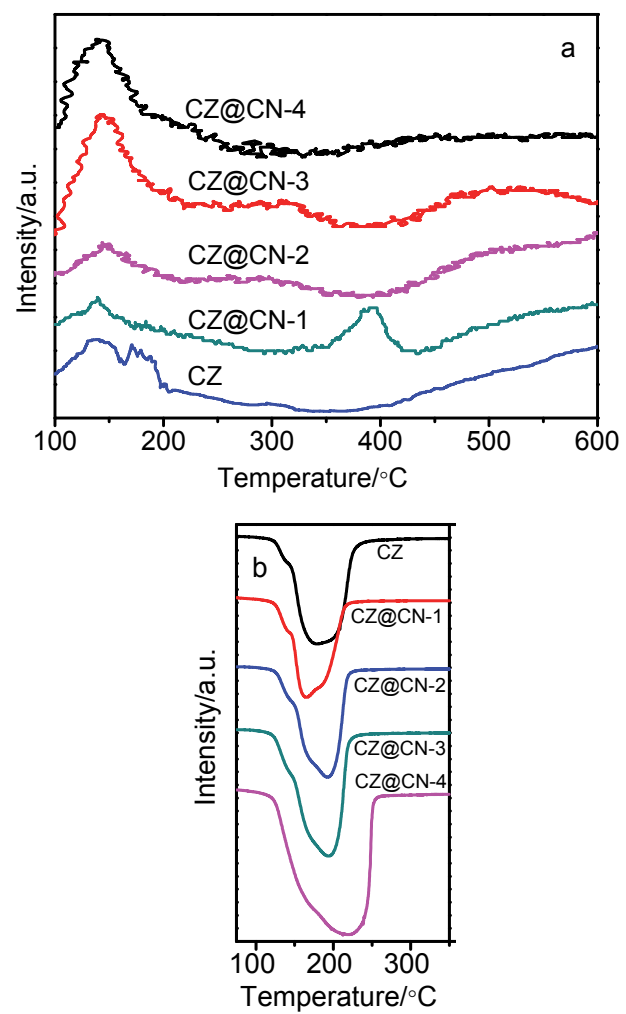

图 5 样品的 $\mathrm{CO}_{2}$ 程序升温脱附曲线(a); 未还原样品的 $\mathrm{H}_{2}-\mathrm{TPR}$ 曲线 图(b)

Figure $5 \mathrm{CO}_{2}$-TPD profiles for the samples (a); $\mathrm{H}_{2}$-TPR profiles of unreduced samples (b)

了弱碱性位，位于 $380{ }^{\circ} \mathrm{C}$ 的脱附峰可以归属为中等强 度的 Lewis 碱性位(MSLB), 位于 $560{ }^{\circ} \mathrm{C}$ 的大峰可以归 属为超强 Lewis 碱性位(SSLB) ${ }^{[18,19]}$. 随着 $\mathrm{CN}$ 含量逐渐 增多，位于 $560{ }^{\circ} \mathrm{C}$ 超强 Lewis 碱性位的峰逐渐减弱并消 失和 $380{ }^{\circ} \mathrm{C}$ 的脱附峰消失, 但 $140{ }^{\circ} \mathrm{C}$ 处 $\mathrm{CO}_{2}$ 脱附峰逐 渐增强, 说明随着 $\mathrm{CN}$ 添加量的增加导致中强碱性位和 超强碱性位消失而弱碱性位逐渐增加. 超强碱性位的消 失是因为 $\mathrm{CN}$ 层过厚导致载体 $\mathrm{ZrO}_{2}$ 表面的碱性位点被 覆盖. 在 CZ@CN-1 样品中出现 Lewis 中等强度碱性位 是由于适当的 $\mathrm{CN}$ 层厚度以及 $\mathrm{CN}$ 层中具有的高电子亲 和性的 $\mathrm{N}$ 物种. 结合催化剂性能测试可知, 中强碱性位 的增加可以增加二乙醇胺脱氢的反应速率，以上结果表 
明适当厚度的 $\mathrm{CN}$ 层引入可以提高催化剂样品 $\mathrm{CZ} @ \mathrm{CN}$ 表面的碱性 ${ }^{[20,21]}$.

\section{$2.6 \mathrm{H}_{2}$ 程序升温还原 $\left(\mathrm{H}_{2}-\mathrm{TPR}\right)$}

图 $5 \mathrm{~b}$ 为五种未还原样品的 $\mathrm{H}_{2}-\mathrm{TPR}$ 图谱. 5 个样品 都在 $160{ }^{\circ} \mathrm{C}$ 和 $175{ }^{\circ} \mathrm{C}$ 附近出现两个低温还原峰, $160{ }^{\circ} \mathrm{C}$ 的还原峰是由催化剂表面高分散的 $\mathrm{CuO}$ 物种还原引起 的, 在 $175{ }^{\circ} \mathrm{C}$ 的还原峰是由晶相 $\mathrm{CuO}$ 的还原引起的. 随 着三聚氧胺的添加量逐渐增多, $160{ }^{\circ} \mathrm{C}$ 和 $175{ }^{\circ} \mathrm{C}$ 的还原 峰逐渐减弱, 样品 $\mathrm{CZ} @ \mathrm{CN}-2$ 和 $\mathrm{CZ} @ \mathrm{CN}-3$ 在 $195{ }^{\circ} \mathrm{C}$ 出 现还原峰, 样品 $\mathrm{CZ} @ \mathrm{CN}-4$ 在 $220^{\circ} \mathrm{C}$ 出现还原峰, 这是由 于进入到 $\mathrm{ZrO}_{2}$ 晶格的 $\mathrm{Cu}^{2+}$ 被还原. 表面低温还原峰随 着三聚氰胺含量的增加逐渐向高温方向移动. 这是因为 $\mathrm{CN}$ 薄层与 $\mathrm{Cu}$ 之间的相互作用, 导致还原温度向高位偏 移. 晶粒尺寸是影响 $\mathrm{CuO}$ 还原的重要因素, 具有较小的 粒径的 $\mathrm{CuO}$ 更容易被还原. 在 $\mathrm{H}_{2}-\mathrm{TPR}$ 表征中往往具有 较低的还原温度, 样品 CZ@CN-1 的还原温度最低, 结 合表 1 可知, CZ@CN-1 具有比 CZ 更小的 $\mathrm{Cu}$ 晶粒尺寸, 分别为 11.4 和 $23.7 \mathrm{~nm}$. 所以样品 CZ@CN-1 催化剂具 有高催化活性, 这与催化剂表面高分散的 $\mathrm{Cu}$ 物质和较 小的 $\mathrm{Cu}$ 粒径尺寸有关.

\section{$2.7 X$ 射线光电子能谱(XPS)}

为了探究样品 $\mathrm{CZ} @ \mathrm{CN}-1$ 中表面 $\mathrm{Cu} 、 \mathrm{Zr} 、 \mathrm{C}$ 、 $\mathrm{N}$ 元素的表面电子状态, 对 $\mathrm{CZ} @ \mathrm{CN}-1$ 催化剂进行了 XPS 表征. 如图 6a 所示, 样品 CZ@CN-1 表面存在着 $\mathrm{Cu} 、 \mathrm{Zr} 、 \mathrm{C} 、 \mathrm{O} 、 \mathrm{~N}$ 五种元素. 图 $6 \mathrm{~b}$ 的 $\mathrm{C} 1$ 高分辨 XPS 图谱中分为 284.6、285.6、288.6、288.9 和 $289.8 \mathrm{eV} 5$ 个峰, 分别归属于 $\mathrm{C}-\mathrm{C}$ 键、 $\mathrm{C}-\mathrm{O}$ 键、 $\mathrm{C}=\mathrm{O}$ 键、 $\mathrm{C}-\mathrm{N}$ 键和 $\mathrm{O}-\mathrm{C}=\mathrm{O}$ 键的峰 ${ }^{[22]}$. 图 $6 \mathrm{c}$ 为样品 $\mathrm{CZ} @ \mathrm{CN}-1$ 的 $\mathrm{N} 1 \mathrm{~s}$ 高分辨 XPS 图谱, 在 $399.8 \mathrm{eV}$ 和 $400.5 \mathrm{eV}$ 处分成两 个峰, 分别为与碳原子以 $\mathrm{H}-\mathrm{N}-(\mathrm{C})_{2}$ 形式相连的吡咯型氮 原子和与三个碳原子相连的石墨氮原子 ${ }^{[23,24]}$, 以上结果 证明 $\mathrm{N}$ 元素成功掺杂进入碳层, 形成了氮掺杂碳层. 图 $6 \mathrm{~d}$ 为 $\mathrm{CZ} @ \mathrm{CN}-1$ 与 $\mathrm{CZ}$ 催化剂的 $\mathrm{Cu} 2 \mathrm{p}$ 高分辨 XPS 图谱, 与 $\mathrm{CZ}$ 催化剂相比, $\mathrm{CZ} @ \mathrm{CN}-1$ 催化剂中 $\mathrm{Cu} 2 \mathrm{p}$ 向高场发 生了移动, 这是由于金属物种与 $\mathrm{CN}$ 层之间的相互作用 导致铜物种的电子向 $\mathrm{CN}$ 层迁移.

\section{8 热重分析(TG)}

图 7 为 $\mathrm{CZ} @ \mathrm{CN}-1$ 与 $\mathrm{CZ}$ 催化剂样品 $\mathrm{TG}$ 图. 由图可 知, $0 \sim 180{ }^{\circ} \mathrm{C}$ 之间两个样品质量都出现下降, 这是因为 催化剂表面水分的脱出. 180 460 ${ }^{\circ} \mathrm{C}$ 之间 CZ@CN-1 催 化剂基本不失重; $\mathrm{CZ}$ 催化剂失重是因为 $\mathrm{ZrO}_{2}$ 层间吸附 水的脱除. $460 \sim 750{ }^{\circ} \mathrm{C}$ 之间两个催化剂的失重主要是 由于结构羟基的脱除引起的, 并且 $\mathrm{CZ} @ \mathrm{CN}-1$ 催化剂失 重速率大于 $\mathrm{CZ}$ 催化剂, 是因为在该范围温度内, $\mathrm{CN}$ 薄 层出现分解. 由于 $\mathrm{CZ} @ \mathrm{CN}-1$ 催化剂用于二乙醇胺脱氢 时, 催化活性高、反应速度极快, 导致反应釜内温度急 剧上升, 但 $\mathrm{CN}$ 层引入像一层保护膜, 在 $460{ }^{\circ} \mathrm{C}$ 高温时
依旧能保护和稳定 CZ@CN 催化剂，防止在高温环境中 $\mathrm{Cu}$ 纳米粒子的烧结, 保持催化剂的活性.
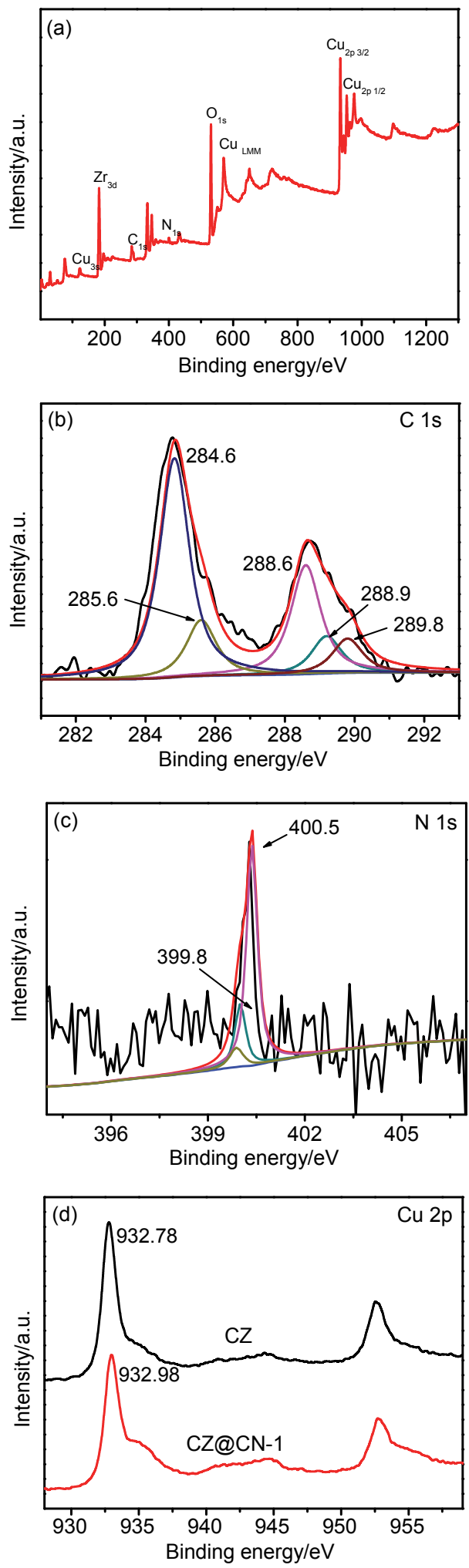

图 6 样品 $\mathrm{CZ} @ \mathrm{CN}-1$ 的全谱(a)、C 1s (b)、N 1s (c) 和 Cu 2p (d) XPS 表征和 $\mathrm{CZ}$ 样品的 $\mathrm{Cu} 2 \mathrm{p}$ (d) XPS 表征

Figure 6 XPS of the survey typical wide (a), C 1s (b), N 1s (c) and Cu $2 p$ (d) for CZ@CN-1 sample and XPS of Cu 2p (d) for CZ sample 


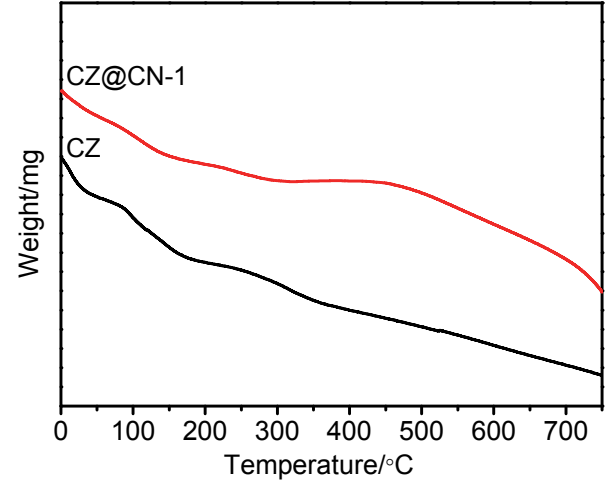

图 7 样品 $\mathrm{CZ}$ 和 $\mathrm{CZ} @ \mathrm{CN}-1$ 的热重曲线

Figure 7 TG proflies of $\mathrm{CZ}$ and $\mathrm{CZ} @ \mathrm{CN}-1$ samples

\section{9 傅里叶红外光谱(FT-IR)}

为了研究催化剂表面组成, 对 CZ@CN-1 和 CZ 催 化剂样品进行傅里叶红外光谱的表征, 如图 8 所示. 催 化剂样品 CZ@CN-1 和 $\mathrm{CZ}$ 在 473、1632、3432 $\mathrm{cm}^{-1}$ 处 均出现了吸收峰, 位于 $3432 \mathrm{~cm}^{-1}$ 处的吸收峰归属于样 品水分子中-OH 的伸缩振动, 位于 $1632 \mathrm{~cm}^{-1}$ 的谱带归 属于样品中-OH 的弯曲振动峰, 位于 $473 \mathrm{~cm}^{-1}$ 的谱带归 属于样品中 $\mathrm{Zr}-\mathrm{O}$ 的特征吸收峰 ${ }^{[25]}$. 样品 $\mathrm{CZ} @ \mathrm{CN}-1$ 还 在 1103 和 $1381 \mathrm{~cm}^{-1}$ 处有吸收峰归属于 $\mathrm{C}-\mathrm{N} 、 \mathrm{C}=\mathrm{N}$ 的伸缩振动峰, 表明在催化剂表面成功地生成了氮掺杂 的碳层. 并且可以发现样品 CZ@CN-1 位于 $1632 \mathrm{~cm}^{-1}$ 的谱带振动强度大于样品 $\mathrm{CZ}$, 表明样品 $\mathrm{CZ} @ \mathrm{CN}-1$ 含 有更多的羟基. 与图 $5 \mathrm{a} \mathrm{CO}_{2}$ 吸脱附曲线所得出的结论 一致.

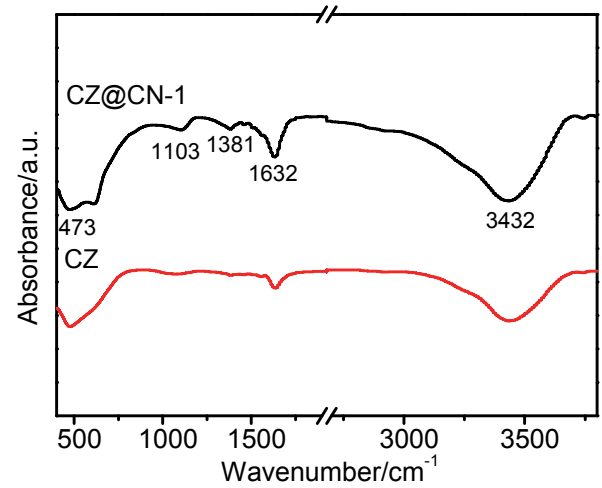

图 8 样品 $\mathrm{CZ}$ 和 $\mathrm{CZ} @ \mathrm{CN}-1$ 傅里叶红外光谱分析图

Figure 8 FT-IR spectra of the $\mathrm{CZ}$ and $\mathrm{CZ} @ \mathrm{CN}-1$ samples

\subsection{0 催化剂脱氢的性能测试}

为了探究样品的催化性能, 将各样品用于二乙醇胺 脱氢的性能测试. 采用高效液相色谱分析法对反应液中 亚氨基二乙酸钠(DSIDA)的含量进行了分析. 表 2 为样 品用于二乙醇胺脱氢合成 DSIDA 的催化性能测试结果. 由表 2 可知, 当以样品 $\mathrm{CZ} @ \mathrm{CN}-1$ 为催化剂时合成的亚
氨基二乙酸钠收率为 $92.8 \%$, 反应时间只需要 $90 \mathrm{~min}$, 初始产气温度降低到 $90{ }^{\circ} \mathrm{C}$, 初次排气时间为反应开始 $25 \mathrm{~min}$; 而 CZ 催化剂反应时间为 $150 \mathrm{~min}$, DSIDA 收率 为 $92.3 \%$, 初始产气温度为 $130{ }^{\circ} \mathrm{C}$, 初次排气时间为反 应开始 $30 \mathrm{~min}$. 随着三聚氧胺添加量逐渐增多, 催化剂 反应时间逐渐延长, 初次排气时间变长, 并且收率逐渐 开始下降, 结合 $\mathrm{H}_{2}-\mathrm{TPR} 、 \mathrm{XRD}$ 和 $\mathrm{CO}_{2}-\mathrm{TPD}$ 测试结果可 知, 是由于 $\mathrm{CN}$ 层的厚度增加使得催化剂表面高分散的 活性铜物种降低, 并且随着三聚氧胺添加量增多, $\mathrm{CN}$ 层 变厚阻碍了催化剂与二乙醇胺的接触以及 $\mathrm{ZrO}_{2}$ 载体的 超强碱性位无法表达, 导致催化剂反应时间增加. 因此 可知在相同催化剂用量的情况下, CZ@CN-1 样品催化 活性最高, 反应时间最短且比 CZ 催化剂缩短 $40 \%$ 的时 间.

多相催化剂的可重复利用性是实际运用的重要性 质, 图 9 为样品 $\mathrm{CZ}$ 和 $\mathrm{CZ} @ \mathrm{CN}-1$ 在二乙醇胺脱氢反应 中循环测试结果. 由图可知, 在相同的反应和循环条件 下, CZ@CN-1 催化剂循环使用八次以后 DSIDA 收率依 然可以达到 88.4\%而 $\mathrm{CZ}$ 催化剂循环使用八次后收率下 降到 75.8\%, 说明 CZ@CN-1 比 CZ 催化剂稳定性好. 这 是因为 $\mathrm{CN}$ 层的包覆成功地稳定了铜纳米粒子, 防止 $\mathrm{Cu}$ 纳米粒子的浸出和氧化. 因此在铜与三聚氧胺物质的量 比为 $4: 1$ 时, 制备的碳氮包覆的 CZ@CN 催化剂活性 高、稳定性好, 可大幅提高对二乙醇胺催化脱氢性能.

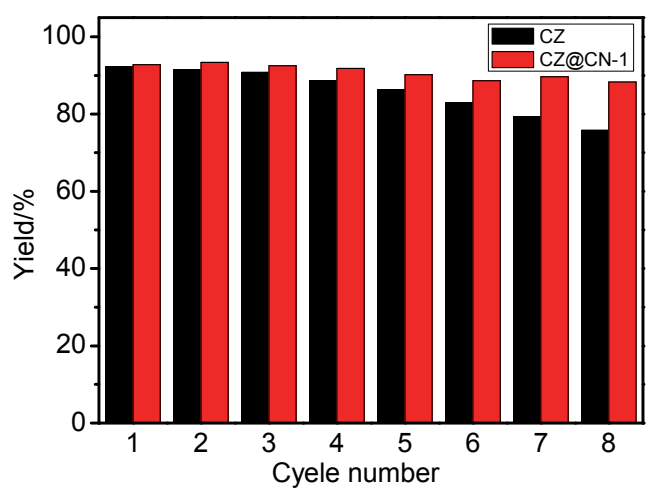

图 9 在二乙醇胺脱氢中, 催化剂样品 $\mathrm{CZ}$ 和 $\mathrm{CZ} @ \mathrm{CN}-1$ 的循环测试 Figure 9 Recyclability of $\mathrm{CZ}$ and $\mathrm{CZ} @ \mathrm{CN}-1$ catalyst in the dehydrogenation of diethanolamine

\subsection{1 催化剂用于二乙醇胺脱氢机理的探讨}

在均相醇类脱氢反应中, 有机碱或碱性化合物常作 为添加剂以加快醇羟基的去质子化过程，从而达到目标 产物的高产率。相似地，在多相催化反应中，碱性载体 可以提高醇类转移脱氢的催化活性. 在 $\mathrm{CZ} @ \mathrm{CN}-1$ 催化 剂中, 即使 $\mathrm{CN}$ 层包覆了铜纳米粒子, 但对二乙醇胺脱 氢仍然有着优异的催化活性. 结合表 1 可知, 这可能与 催化剂的比表面积有关, CZ@CN-1 催化剂比表面积比 $\mathrm{CZ}$ 催化剂大. 值得注意的是, 与其他催化剂样品相比, 
$\mathrm{CZ} @ \mathrm{CN}-1$ 具有中强碱性位点, 且 CZ@CN-1 表现出更 优异的二乙醇胺脱氢性能. 所以 $\mathrm{CZ} @ \mathrm{CN}-1$ 催化剂有较 高的二乙醇胺转移脱氢活性可能与其表面碱性位点有 关.

在多相醇类脱氢反应中, 通常认为二乙醇胺催化脱 氢制备亚氨基二乙酸与一乙醇胺催化脱氢制备甘氨酸 的过程机理类似 ${ }^{[26]}$, 即二乙醇胺在 $\mathrm{Cu}$ 基催化剂的作用 下生成亚氨基二乙醛, 两分子的醛在强碱的环境下转化 成亚氨基二乙酸钠. 在当前 $\mathrm{CZ} @ \mathrm{CN}$ 作为催化剂的体系 中, 应是二乙醇胺上的羟基与 $\mathrm{Cu}$ 相邻的表面强碱性位 以及 $\mathrm{Cu}$ 与 $\mathrm{CN}$ 层相互作用下形成的中强碱性位点发生 键合从而被夺取质子, 因此和 $\mathrm{Cu}$ 物种形成醇盐中间体, 碱性溶液可以加快去质子化的速度 ${ }^{[27]}$. 醇盐中间体被 吸附在 $\mathrm{Cu}$ 上, 随后另一个活性质子在 $\mathrm{Cu}$ 的催化作用下 以 $\alpha-\mathrm{H}$ 断裂的方式被脱除, 最终形成醛类物质. 由醛制 备酯的反应过程可能有两种反应路线: (1)两分子醛发生 康尼扎罗反应; (2)醛和醇发生亲核加成反应. 但是由第 一种路线无法得到对应的酯, 当醛中有 $\alpha-\mathrm{H}$ 时会被反应 物中的碱拿走发生羟醛缩合反应, 所以本反应不会按照 康尼扎罗反应路线进行. 第二条路线过程为: 碱夺取了 中间产物醛上的 $\alpha-\mathrm{H}$, 因此导致亲电性的 $\alpha-\mathrm{C}$ 出现, 从 而与具有亲核活性的醇盐反应生成酯，酯在碱性环境下 分解得到亚氨基二乙酸盐 ${ }^{[28]}$.

\section{3 结论}

本文以三聚氰胺为碳源和氮源, 采用简单的热分解 制备出具有核壳结构氮掺杂碳层包覆的 $\mathrm{Cu}-\mathrm{ZrO}_{2}$ 催化 剂, 并将其应用于二乙醇胺的液相转移脱氢反应中. 在 铜与三聚氰胺物质的量比为 $4: 1$ 时所制备的 CZ@CN-1 催化剂表现出较高的二乙醇胺催化脱氢活性. 一方面, 与 $\mathrm{CZ}$ 催化剂相比, CZ@CN-1 催化剂比表面积大, 颗粒 尺寸更小, 表面具有高分散 $\mathrm{Cu}$ 纳米粒子; 另一方面, $\mathrm{CN}$ 层中高电子亲和力的 $\mathrm{N}$ 所产生的表面 SLB 碱性位与 SLB 位接触的 $\mathrm{Cu}$ 纳米粒子产生相互协同作用, 使催化 剂含有更多的碱性位点, $\mathrm{Cu}$ 纳米粒子具有更好的分散 性, 构建出新的纳米环境, 是提高二乙醇胺脱氢反应效 率的关键. 同时, $\mathrm{CN}$ 层与 $\mathrm{Cu}$ 纳米粒子的相互作用能够 稳定铜纳米颗粒, 防止铜物种在反应过程中的浸出和生 长从而具有很好的稳定性. 但是随着三聚氧胺量的添 加, $\mathrm{CN}$ 层逐渐变厚阻碍 $\mathrm{CZ}$ 催化剂本身与二乙醇胺的接 触和 $\mathrm{ZrO}_{2}$ 表面强碱性位的表达, 从而降低催化活性. 并 通过对反应机理的探讨, 否定了二乙醇胺脱氢制备亚氨 基二乙酸钠过程中发生康尼扎罗反应，提出醛和醇发生 亲核加成反应的途径. 本研究提供了一种简单的方法, 制备具有氮掺杂碳包覆的 $\mathrm{Cu}-\mathrm{ZrO}_{2}$ 催化剂, 为后续制备 高催化脱氢活性且稳定性好、分散性好的 $\mathrm{Cu}$ 基催化剂 提供新的思路.

\section{4 实验}

\section{1 实验药品与仪器}

硝酸铜 $\left(\mathrm{Cu}\left(\mathrm{NO}_{3}\right)_{2} \cdot 3 \mathrm{H}_{2} \mathrm{O}\right)$, 广东汕头市西陇化工厂; 氯氧化锆 $\left(\mathrm{ZrOCl}_{2} \bullet 8 \mathrm{H}_{2} \mathrm{O}\right)$, 上海阿拉丁生化科技股份有 限公司; 氢氧化钠 $(\mathrm{NaOH})$, 天津市光复科技发展有限公 司; 二乙醇胺(DEA), 广东汕头市西陇化工厂; 三聚氰 胺 $\left(\mathrm{C}_{3} \mathrm{H}_{6} \mathrm{~N}_{6}\right)$, 广东汕头市西陇化工厂; 去离子水 $\left(\mathrm{H}_{2} \mathrm{O}\right)$, 实验实自制; 工业氢气(纯度 $>99.9 \%)$ ，工业氮气(纯度 $>99.9 \%$ ).

\section{2 实验步骤}

称取 $6.44 \mathrm{~g} \mathrm{ZrOCl}_{2} \bullet 8 \mathrm{H}_{2} \mathrm{O} 、 2.42 \mathrm{~g} \mathrm{Cu}\left(\mathrm{NO}_{3}\right)_{2} \bullet 3 \mathrm{H}_{2} \mathrm{O}$ 以 及一定质量的 $\mathrm{C}_{3} \mathrm{H}_{6} \mathrm{~N}_{6}$ 溶于 $600 \mathrm{~mL}$ 蒸馏水中, 搅拌使其 完全溶解, 并得到蓝色混合盐溶液; 称取 $4.8 \mathrm{~g}$ 氢氧化钠 溶于 $250 \mathrm{~mL}$ 水中, 然后逐滴加入蓝色混合盐溶液中, 使其全部沉淀后再继续搅拌 $6 \mathrm{~h}$. 将溶液静置老化过夜 后, 用蒸馏水洗涤沉淀物至上清液无氯离子及 $\mathrm{pH}$ 值等 于 7. 将所得滤饼置于 $60{ }^{\circ} \mathrm{C}$ 烘箱内过夜干燥. 将干燥所 得固体研磨成粉, 置于箱式气氛炉中, 在 $\mathrm{N}_{2}$ 氛围中 $550{ }^{\circ} \mathrm{C}$ 裧烧 $4 \mathrm{~h}$, 得 $\mathrm{CuO} / \mathrm{ZrO}_{2} @ \mathrm{CN}$ 前驱体. 将得到的 $\mathrm{CuO} / \mathrm{ZrO}_{2} @ \mathrm{CN}$ 前驱体放入管式炉中, 在 $\mathrm{H}_{2}$ 氛围下, 以 $3{ }^{\circ} \mathrm{C} / \mathrm{min}$ 的升温速率至 $350{ }^{\circ} \mathrm{C}$, 并在该温度下还原 $4 \mathrm{~h}$, 自然降温冷却得到 $\mathrm{Cu} / \mathrm{ZrO}_{2} @ \mathrm{CN}$ 催化剂.

\section{3 表征}

FEI Tecnai G2 F20 型透射式电子显微镜(TEM, 美 国 FEI 公司); SU8200 型场发射扫描电镜(SEM, 日本日 立公司); TGA-50 型热分析仪(美国 TA 公司); TriStar II 3020 型比表面积和孔径分析仪(BET, 美国麦克默瑞提 克仪器有限公司); AutoChem II 2920 型全自动程序升温 化学吸附仪 $\left(\mathrm{H}_{2}-\mathrm{TPR} 、 \mathrm{CO}_{2}-\mathrm{TPD}\right.$ 麦克默瑞提克仪器有限 公司); NICOLET 380 型傅里叶红外光谱仪(FTIR，美国 尼高力公司); Dmax-2500/PC 型 $\mathrm{X}$ 射线多晶粉末衍射仪 (XRD，日本理学株式会社)；1200 型高效液相色谱仪(美 国安捷伦公司).

\section{4 催化剂脱氢实验}

将 $10 \mathrm{~g}$ 二乙醇胺、 $2 \mathrm{~g}$ 催化剂、 $8.5 \mathrm{~g}$ 氢氧化钠 (溶 于 $80 \mathrm{~mL}$ 去离子水)加入至高压反应釜中. 检查反应釜 气密性，通入 $\mathrm{N}_{2}$ 排空 $5 \sim 6$ 次后加压至 $1 \mathrm{MPa}, 4{ }^{\circ} \mathrm{C} / \mathrm{min}$ 的升温速率下, 升温至 $160{ }^{\circ} \mathrm{C}$, 在转速为 $400 \mathrm{r} / \mathrm{min}$ 进行 搅拌, 用转子流量计计量排出的气体体积. 当气压升高 至 $1.5 \mathrm{MPa}$ 后打开排气阀, 降压至 $1 \mathrm{MPa}$ 后关闭排气阀, 并记录每次排气的体积和排气温度. 反应过程中反应釜 内压力在 $20 \mathrm{~min}$ 内无变化即视为反应结束. 采用液相色 谱进行定量分析，用强阴离子交换色谱柱(Hypersil $\mathrm{SEX}, 5 \mu \mathrm{m}, 4.6 \mathrm{~mm} \times 250 \mathrm{~mm})$, 从而计算产物亚氨基二 乙酸钠的收率. 


\section{References}

[1] Zhu, Y.; Kong, X.; Li, X.; Ding, G.; Zhu, Y.; Li, Y. W. ACS Catal. 2014, 4, 3612.

[2] Duan, Z. K.; Li, S.; Xie, F.; Yan, J. H.; Zhang, T. Chem. Res. Appl. 2015, 27, 417. (段正康, 李晟, 谢帆, 问建华, 张涛, 化学研究与 应用, 2015, 27, 417.

[3] Tang, Q. L.; Liu, Z. P. J. Phys. Chem. 2010, 114, 8423.

[4] Agrell, J.; Birgersson, H.; Boutonnet, M.; Melián-Cabrera, I.; Navarro, R. M.; Fierro, J. L. G. J. Catal. 2003, 219, 389.

[5] Huo, J. P.; Song, H. H.; Chen, X. H.; Zhao, S. Q.; Xu, C. M. Carbon Techniques. 2006, 25, 22. (霍俊平, 宋怀河, 陈晓红, 赵锁奇, 徐 春明，炭素技术, 2006, 25, 22.)

[6] Liu, J. Y.; Yang, P. J.; Zhang, J. F.; Ma, S. J. Petrochem. Technol. 2004, 33，330. (刘金河, 杨普江, 张景峰, 马淑杰, 石油化工, 2004, 33, 330.)

[7] Li, H. T.; Chen, H. R.; Zhang, Y.; Gao, C. G.; Zhao, Y. X. Chinese J. Catal. 2011，32，111. (李海涛，陈昊然，张因，高春光，赵永祥， 催化学报, 2011, 32, 111.)

[8] Roy, R. K.; Lee, K. J. Biomed. Mater. Res. B 2010, 83B, 72.

[9] Zhang, Z. Q.; Ge, C. X.; Chen, Y. G.; Wu, Q.; Yang, L. J.; Wang, X. Z.; Hu, Z. Acta Chim. Sinica 2019, 77, 60. (张志琦, 葛承宣, 陈玉 刚, 吴强, 杨立军, 王喜章, 胡征, 化学学报, 2019, 77, 60.)

[10] Su, D. S.; Zhang, J.; Frank, B.; Thomas, A.; Wang, X.; Paraknowitsch, J.; Schlögl, R. ChemSusChem 2010, 3, 169.

[11] Mabena, L. F.; Sinha Ray, S.; Mhlanga, S. D.; Coville, N. J. Appl. Nanosci. 2011, 1, 67.

[12] Dai, X. Q.; Zhu, Y. B.; Xu, X. L.; Wen, J. Q. Chin. J. Org. Chem. 2017，37，577. (戴小强, 朱亚波, 许孝良, 翁建全, 有机化学,
2017, 37, 577.)

[13] Watanabe, H.; Asano, S.; Fujita, S.; Yoshida, H.; Arai, M. ACS Catal. 2015, 5, 2886.

[14] Zhang, P.; Wang, Q. N.; Yang, X.; Wang, D.; Li, W. C.; Zheng, Y. P.; Chen, M. S.; Lu, A. H. ChemCatChem 2017, 9, 505.

[15] Shi, R. N.; Zhao, J. X.; Liu, S. S.; Sun, W.; Li, H. X.; Hao, P. P.; Li, Z.; Ren, J. Carbon 2018, 130, 185.

[16] Wen, Z.; Liu, J.; Li, J. Adv. Mater. 2008, 20, 743.

[17] Unnikrishnan, P.; Srinivas, D. Ind. Eng. Chem. Res. 2012, 51, 6356.

[18] Hu, Q.; Yang, L.; Fan, G. L.; Li, F. Chem. Nano. Mat. 2016, 2, 888.

[19] Wang, J.; Lei, Z.; Qin, H.; Zhang, L.; Li, F. Ind. Eng. Chem. Res. 2011, 50, 7120

[20] Hu, Q.; Fan, G.; Yang, L.; Cao, X.; Zhang, P.; Wang, B.; Li, F. Green Chem. 2016, 18, 2317.

[21] Xu, J.; Shen, K.; Xue, B. J. Mol. Cayal. A 2013, 372, 105.

[22] ABUDUHEIREMU, Awati; Zhang, D. D.; HALIDAN, Maimaiti Chem. J. Chin. Univ. 2019, 40, 306. (阿卜杜黑热木・阿瓦提，张得 栋, 哈丽丹 - 买买提, 高等学校化学学报, 2019, 40, 306.)

[23] Chen, S.; Bi, J.; Zhao, L.; Yang, C.; Ma, Y.; Wu, Q.; Wang, X.; Hu, Z. Adv. Mater. 2012, 24, 5593.

[24] Sharitfi, T.; Hu, G.; Jia, X.; Wagberg, T. ACS Nano 2012, 6, 8904.

[25] Wang, X. X.; Zhang, L. H.; Lin, H. J.; Nong, Q. Y.; Wu, Y.; Wu, T. H.; He, Y. M. RSC Adv. 2014, 4, 40029

[26] Yang, Y.; Duan, Z.; Liu, W. Chem. Reac. Eng. Technol. 2001, 17, 210.

[27] Balaraman, E.; Khaskin, E.; Leitus, G.; Milstein, D. Nat. Chem. 2013, 5, 122 .

[28] Neurock, M.; Tao, Z.; Chemburkar, A.; Hibbitts, D. D.; Lglesia, E. Faraday Discuss. 2017, 197, 181. 